\title{
Flash Video in College English Education
}

\author{
Ying Chen ${ }^{1, \mathrm{a}}$
}

\author{
${ }^{1}$ Harbin Institute of Technology (Weihai), Shandong, China \\ ${ }^{a}$ chenyinghhh@126.com
}

\begin{abstract}
Flash Video, a widely used new stream video format today, is small-sized, easy to play, and transformable from other format videos. It has overcome the shortcomings like inconvenience in operation, high bandwidth occupation and large file size. This paper focuses on the current problems in video playing, the technical advantages of flash video, the transformation and application of Flash video.
\end{abstract}

Keywords: stream media, flash video, college English education

\section{Flash Video 在大学英语视频教学中的应用}

陈莹 ${ }^{1, a}$

${ }^{1}$ 哈尔滨工业大学 (威海), 山东威海, 中国
${ }^{a}$ chenyinghhh@126.com

\section{摘要}

Flash Video 是一种新的流媒体格式, 目前得到了广泛应用, 具有易于播放、生成文件小、易于转换等 优点, 可以很好地克服目前视频教学平台中存在的视频格式多、不易播放、占用带宽高、网络堵塞、 占用存储空间多等缺点。本文主要对视频播放目前存在的问题、Flash Video 的技术优点、Flash Video 的转换生成及具体应用等进行探讨。

关键词: 流媒体, Flash Video, 大学英语教育

\section{1. 引言}

随着计算机多媒体技术的发展, 视频教学得到了越来越多 的应用, 成为传统教学方式的一个很好的补充和延伸。一 个好的视频学习网络平台可以提高学生的学习兴趣, 自主 选择与自身相适应的内容, 自由选择学习时间, 因此很值 得推广。而一个好的视频学习平台除了要有丰富和优质的 学习内容, 易用性和可用性则显得更为重要, 因为如果不 能顺畅的使用, 则内容就显得没有意义。

\section{2. 视频教学目前存在的问题}

尽管目前很多高校都提供了视频学习网络平台, 但由于以 下方面的原因，或多或少的限制了应用。

\section{1. 视频格式多}

自从出现了计算机多媒体技术之后, 视频编码出现了百家 争鸣的现象, 各大公司争相推出了自己的视频编码格式, 如微软的 avi、asf、wmf, 苹果公司的 mov 格式, real networks 公司的 rm、rmvb 格式, 以及国际标准 mpeg 等。 为了能播放这些不同格式的视频文件, 就需要不同类型的 播放软件。当把视频放到网络上在线观看时, 就需要为汶 览器安装播放插件, 为了能看多种格式的视频, 就需要安 装多个插件。若没有安装或安装不正确或版本不正确将不
能播放。

\section{2. 带宽不足}

由于目前高校的经费普遍比较紧张, 限制了学校在网络方 面的投入。视频播放需要很高的带宽, 若带宽有限而使用 者众多, 将会出现不能播放或播放不连续的现象, 影响了 学习效果。

\section{3. 存储空间不足}

由于视频文件占据的存储空间比较大, 随着存放的视频文 件不断增加, 服务器的存储空间显得越来越紧张。尽管可 以增加硬盘来扩充存储空间, 但如果服务器的硬盘扩展槽 已经插满, 则无法通过增加硬盘的方式来增加空间, 只能 增加或更换服务器, 这无疑增加了预算, 而且经常不太现 实。

\section{4. 服务器响应慢}

由于播放视频对系统资源占用很大, 因此若同时在线观看 的人数多, 就会超过服务器的负载能力, 出现响应延时的 现象，造成观看者的等待。

工欲善其事，必先利其器。应用一个好的技术，有时可以 起到事半功倍的效果。对于上述几个问题，应用 Flash Video 技术可以得到极大的缓解。因为 Flash Video 播放简 


\section{1. 易于使用}

单, 文件体积小, 减少了带宽和存储空间的占用, 降低了 服务器的负载, 因此可以提高服务器的响应能力, 让视频 可以得到流畅的播放。

\section{Flash Video 技术介绍}

Flash Video（简称 flv）是一种新的流媒体视频格式, 是 macromedia 公司（现已被 Adobe 公司收购）随着 Flash 动画制作软件 Flash MX 的推出而发展起来的一种视频格 式。虽然推出较晚, 但由于其形成的文件小, 加载速度快 等优点使其成为近年来增长最快、应用最为广泛的视频传 播格式。目前国内的视频博客平台土豆网、被 google 斥 资 16.5 亿美元收购的视频博客平台 youtube 网、香港的凤 凰网, 都是采用 flash video 格式进行视频的传播。Flash Video 格式主要有如下优点。

表 1 其它视频格式与 $f 1 v$ 格式的比较

\begin{tabular}{|l|l|l|l|l|l|l|}
\hline 媒体格式 & $\begin{array}{l}\text { 原文件大 } \\
\text { 小 }(\mathrm{KB})\end{array}$ & $\begin{array}{l}\text { 播放 时 } \\
\text { 间 }\end{array}$ & $\begin{array}{l}\text { 中等质量大小 } \\
(\mathrm{KB})\end{array}$ & $\begin{array}{l}\text { 每分钟数 } \\
\text { 据量(KB) }\end{array}$ & $\begin{array}{l}\text { 低 质 量 小 小 } \\
(\mathrm{KB})\end{array}$ & $\begin{array}{l}\text { 每分钟数 } \\
\text { 据量 }(\mathrm{KB})\end{array}$ \\
\hline VCD & $114128 \mathrm{~K}$ & $11: 01$ & $42627(37 \%)$ & 3880 & $16753(15 \%)$ & 1500 \\
\hline $\begin{array}{l}\text { DVD (分 } \\
\text { 辨率 } \\
\text { 不变) }\end{array}$ & 375042 & $5: 53$ & $40334(11 \%)$ & 6860 & $15824(4 \%)$ & 2700 \\
\hline $\begin{array}{l}\text { DVD (转 } \\
\text { 为 VCD } \\
\text { 分辨率) }\end{array}$ & 375042 & $5: 53$ & $16083(4 \%)$ & 2730 & $6447(1.7 \%)$ & 1100 \\
\hline MP3 & 6550 & $4: 24$ & $2227(34 \%)$ & 500 & $1193(18 \%)$ & 270 \\
\hline
\end{tabular}

可以看出, flv 格式文件在大小方面有很大的优越性。生 成的 flv 文件大小与原媒体文件的画面质量紧密相关, 画 质越好, 可以接受的 flv 文件就可以生成的越小, 所以同 样时间长度的 DVD 格式的文件生成的 flv 文件可以比 VCD 格式的文件生成的 flv 文件更小。根据笔者观察, 凤 凰网视频播放使用的分辨率为 400X300, 画面质量优异, 声音清晰。但每分钟数据量只为 $2.7 \mathrm{M}$ 左右, 半个小时的 数据量仅为 $80 \mathrm{M}$ 左右。可见使用 flash video 可以在很低 的网络数据传输量下得到很好的观看效果。

\section{3. 易于生成}

既然 flash video 有易于播放和文件小的优点, 有没有办法 把其他格式的视频文件转换为 flv 格式呢? 为了推广 flv 格式, Adobe 公司提供了转换工具, 可以将很多种其他格 式的视频文件, 如 asf,avi,mov,mp4,mpg,wmv 等转换为 flv 格式。即使有些格式不能转换, 如比较流行的 $\mathrm{rm}, \mathrm{rmvb}$ 格式, 也可以找到第三方提供的转换工具进行转换, 如 Ultra Flash Video FLV Converter。

\section{4. 流式播放}

Flv 是为播放网络视频而诞生的格式, 因此它支持流式播 放, 可以边下载边播放, 不用等待整个视频文件全部传送 完成。
Flv 格式是因为 flash 的普及而迅速发展起来的。Flash player 目前在全世界计算机上的普及率达到 $98.8 \%$, 这是 迄今为止市场占有率最高的软件产品。Flv 不需要特殊的 播放器, 只要计算机上安装有 flash player, 就可以观看 flv 视频, 因此绝大多数的计算机都可以观看 flv 视频。

\section{2. 生成文件小}

Flv 格式的视频文件体积很小，下面以 VCD、DVD、MP3 格式为例, 将其转换为 flv 格式时的文件大小和百分比在 下表中表示出来:

\section{5. 跨平台}

即使 windows 操作系统占据了市场主流, 但仍然有一部 分人使用的不是 windows 操作系统, 而是 linux 等其他操 作系统。对于某些格式的视频文件在非 windows 操作系 统上观看存在一定的困难, 但由于 flash 技术在各种操作 系统上都得到了很好的支持, 因此 flv 文件可以在这些系 统上得到很好的播放。

\section{4. 视频转换操作}

目前 flv 视频主要是从其他格式的视频转换过来的, 根据 实际需要, 可以在转换之前对画质、音质、分辨率大小等 参数进行设置。下面将介绍两款常用的转换软件的使用方 法。

\subsection{Flash Video Encoder}

Flash Video Encoder 是 Adobe 公司自己的转换软件。在安 装 Adobe Flash 这款设计软件时会附带安装 Flash Video Encoder, 目前最高版本为 CS3。Flash Video Encoder 可以 对目前大多数的视频格式进行转换, 除了 $\mathrm{rm}$ 和 $\mathrm{rmvb}$, 因 为这两种格式有 realnetworks 公司的版权。该软件界面简 单, 易于操作。启动后, 界面如图 1 所示。 


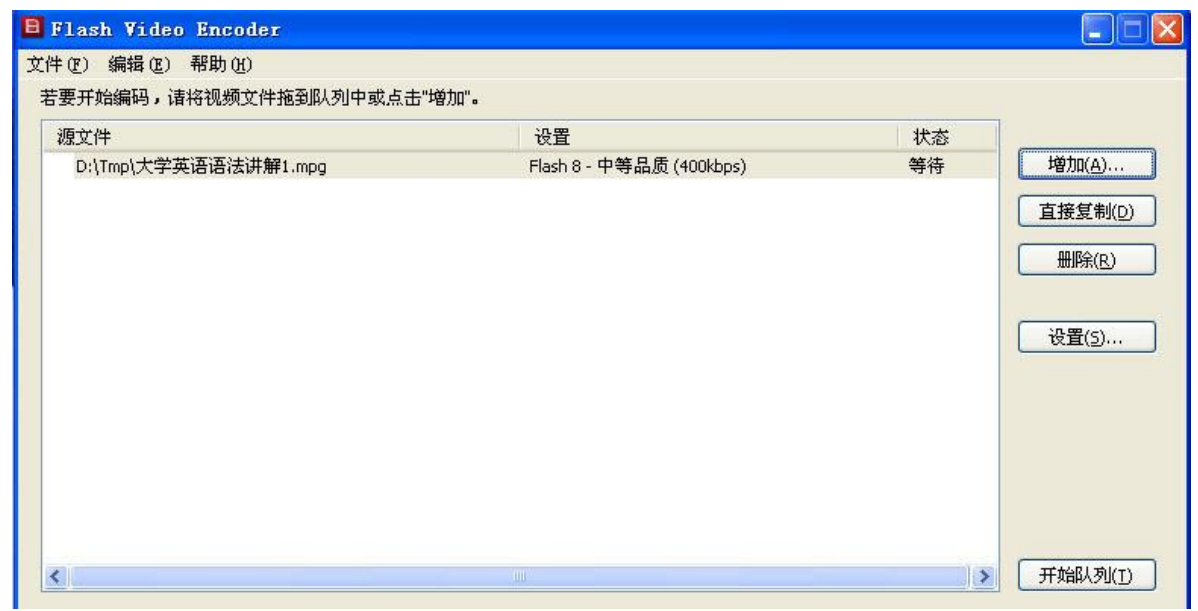

图 1 Flash Video Encoder 操作界面

首先点击 “添加” 按钮添加要转换的文件。从弹出的打开 文件对话框中选择要转换的文件。然后点击 “设置” 按钮, 选择一个 flash 视频编码配置文件。根据需要选择高品质、 中等品质或低品质的编码方案。除了选择预设的编码方 案, 还可以对视频、音频、分辨率进行更进一步的设置。 视频可以设置的主要选项有图像品质的高中低, 最大数据 传输率，帧频等。

音频可以设置音频的数据速率, 一般为 $32 \mathrm{kbps}-96 \mathrm{kbps}$ 之 间。因为网络视频不需要很高的分辨率, 如果原视频的分 辨率比较高, 可以在此设为比较低的分辨率。设置完成后, 点击开始队列按钮就开始了转换过程。转换完成后, 就在

同目录下生成了同名的 flv 文件。

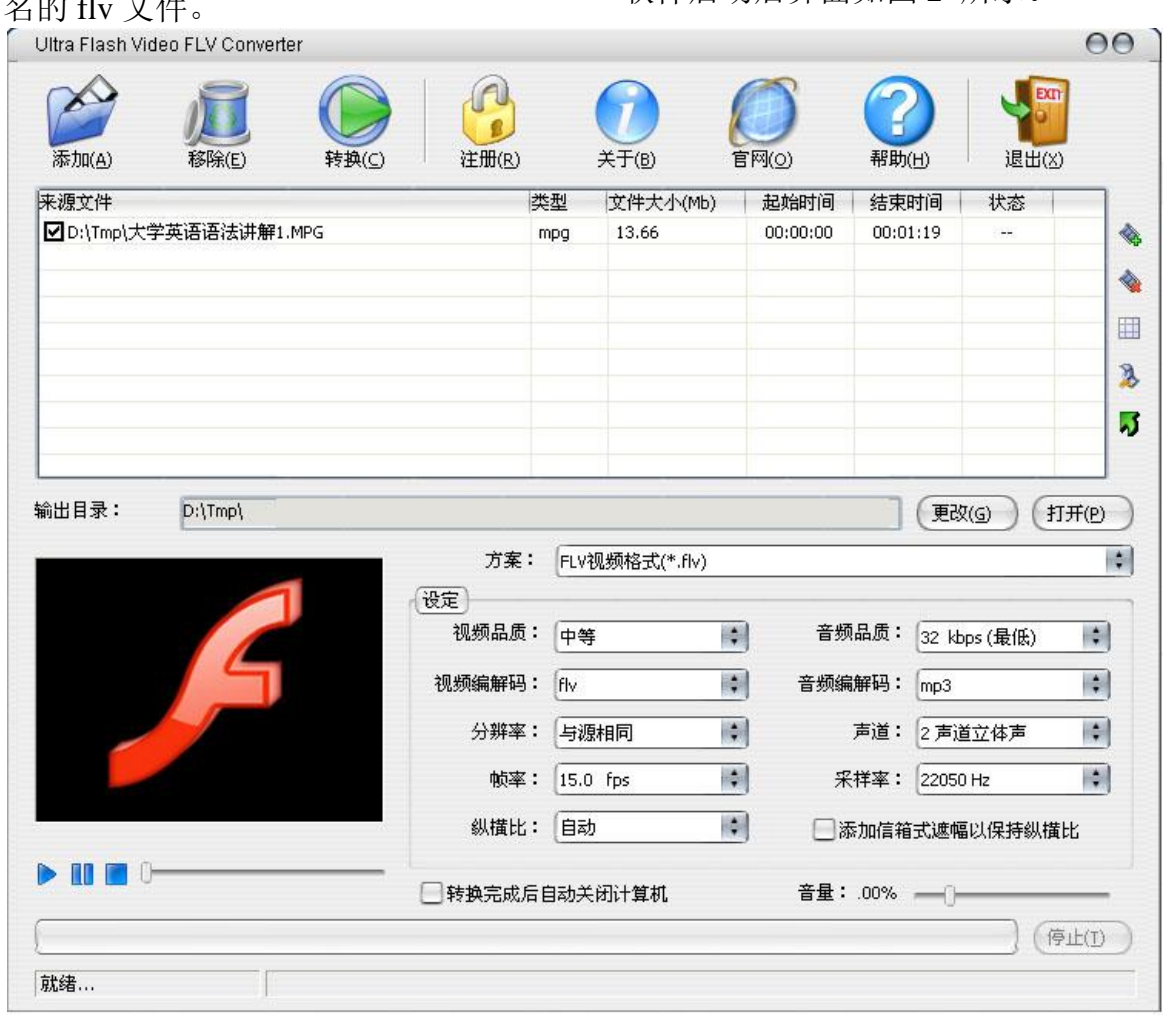

图 2 Ultra Flash Video FLV Converter 操作界面

\subsection{Ultra Flash Video FLV Converter}

除了 Adobe 公司的 Flash Video Encoder, 还有一款非常不 错的第三方软件 Ultra Flash Video FLV Converter，该软件 几乎可以将所有视频格式转换为 flv 格式。该软件的主页 为

http://www.aone-soft.com/flash_video_flv_converter.htm。 该软件也易于使用，所有设置都在一个画面中，也可以对 视频品质、音频品质、分辨率、帧频等参数进行设置。该 软件启动后界面如图 2 所示。

\section{3. $M P 3$}

在很多时候, 我们只需要在网上播放音频而不需要播放视 频, 为了易于播放, 我们仍然可以将其转换为 flv 文件, 10 分钟的音频只需 2-3M 存储空间。转换 mp3 文件时, 
计工具 Dreamweaver CS3 将其加入到网页中进行播放。 下面介绍利用 Dreamweaver 将 flv 视频文件加入到网页中 的方法。

新建或打开一个 html 网页, 选择菜单 “插入记录一〉媒体

-> Flash 视频”, 将弹出插入 Flash 视频对话框。

\section{Flash Video 的发布}

Flv 文件准备好后, 我们就可以利用 Adobe 公司的网页设

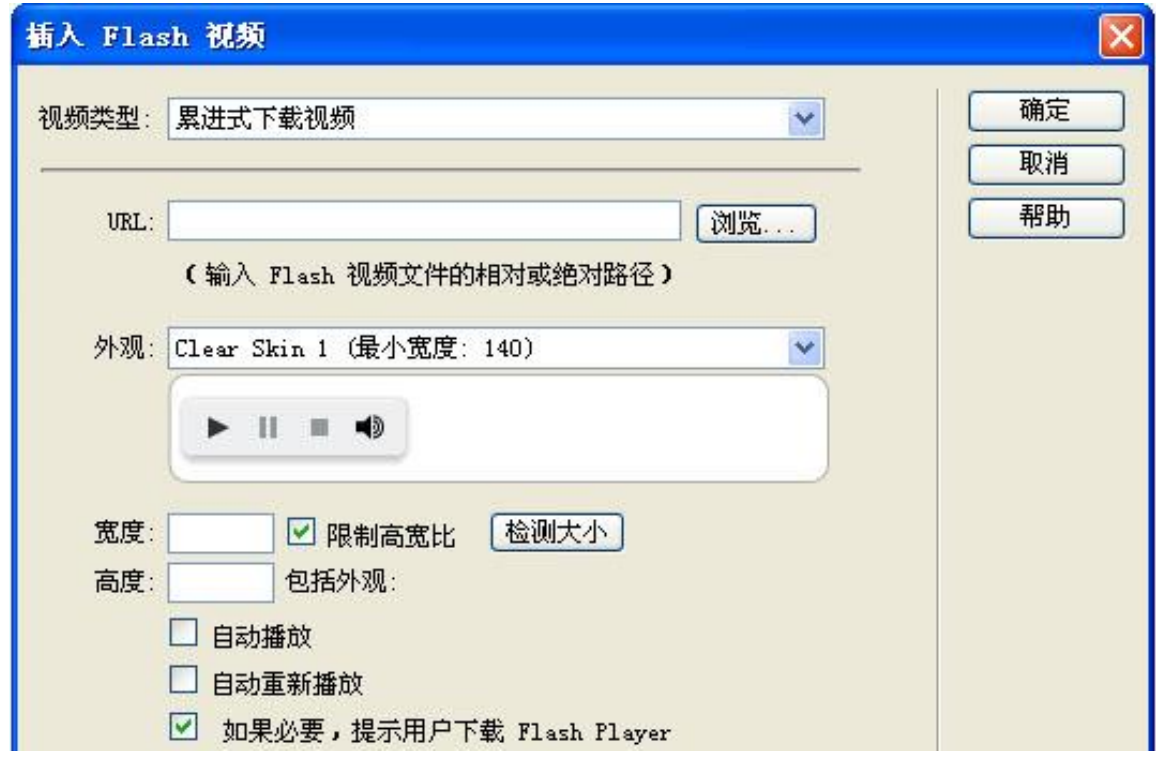

图 3 利用 Dreamweaver 插入 flv 视频

对话框中主要参数的定义如下:

- 视频类型：选择 “累进式下载视频”, 与传统的 “下载并播放”视频传送方法不同, “累进式下 载”允许在下载完成之前就开始播放视频文件, 大大减少等待时间。

- URL: 指定 FLV 文件的相对路径或绝对路径。 若 要 指 定相 对 路径 ( 例 如, mypath/myvideo.flv), 请单击 “浏览” 按钮, 导航到 FLV 文件并将其选定。若要指定绝对 路径, 请键入 FLV 文件的 URL（例如, http://www.example.com/myvideo.flv)。

- 外观：指定 Flash 视频组件的外观。所选外观 的预览会出现在 “外观” 弹出菜单下方。该外 观包含播放、暂停、停止、音量调节等控制功 能。

- 宽度: 以像素为单位指定 FLV 文件的宽度。 若要让 Dreamweaver 确定 FLV 文件的准确 宽度，请单击 “检测大小” 按钮。

- 高度: 以像素为单位指定 FLV 文件的高度。 若要让 Dreamweaver 确定 FLV 文件的准确 高度, 请单击 “检测大小” 按钮。

- 自动播放: 指定在 Web 页面打开时是否播放 视频。

- 自动重新播放：指定播放控件在视频播放完之 后是否返回起始位置。

- 提示用户下载 Flash Player: 在页面中插入代 码, 该代码将检测查看 Flash 视频所需的 Flash Player 版本, 并在用户没有所需的版本时 提示其下载 Flash Player 的最新版本。

\section{6. 具体应用}

有了 flash video 这种方便易用的轻量级的视频格式, 可以 让我们在很多方面进行教学方面的应用。

\section{1. 听力训练}

将 MP3 听力材料转换为 flv 文件后, 放在网上, 并进行 相应的分类。网页上不仅可以播放录音, 也可以显示录音 的文本，供学生检验理解的正确性。

\section{2. 名师课堂}

将已经录制的一些教学录像和教程进行转换后, 放在网 上, 可以让学生自主的选择内容, 极大的提高学习的自主 性和学习效果。如语法教程、四六级讲座等教师自己录制 的课程。

\section{3. 精品教程}

目前有很多种类的英语学习教程, 有 VCD 版的也有 DVD 版的, 如走遍美国、空中英语教室等。如果直接把它们放 在网上一方面占用空间大另一方面也不适合直接观看。如 果把它们转换成小巧的 flv 文件, 既可以大幅减少空间占 用又能流畅的播放。尽管视觉效果有所降低, 但完全可以 满足以学习为目的的需要。 


\section{4. 影视欣赏}

可以将一些经典的影视作品或片断转换为 flv 格式供学生 欣赏, 以此提高视听能力。由于一部电影只需 $200 \mathrm{M}$ 左右 的空间, 可以极大地降低存储需求。

\section{5. 英文歌曲欣赏}

通过欣赏和学习英文歌曲, 也可以加深对英美文化的理 解。通过将一些精美歌曲的 MP3、MTV 片断转换为 flv 格式放在网页上, 并提供歌词, 可以提高学生参与的兴趣, 提升网络的人气。

\section{7. 结语}

多媒体网络视频教学在现代英语教学中发挥了越来越重 要的作用, 但经常会受到存储空间、带宽、服务器响应等 方面的制约。Flash video 的诞生为我们提供了一个很好的 解决方案。可以在不增加硬件投资的前提下, 很好的解决 我们遇到的难题。

\section{致谢}

本文受到哈尔滨工业大学 (威海) 语言文学学院 2017 年 教学研究立项资助项目 “基于隐性课程理念的大学英语 C 级班课程建设” (YYWX201718) 的资助, 项目起止时间: 2017.03-2019.02

\section{参考文献}

[1] Tom Green, David Stiller. Foundation Flash CS3 for Designers, Apress Company, 2007.

[2] Joseph Lowery. Dreamweaver CS3, Wiley Publishing, 2007. 